\title{
Statistics and fluctuation theorem for boson and fermion transport through mesoscopic junctions
}

\author{
Upendra Harbola, ${ }^{1}$ Massimiliano Esposito, ${ }^{1,2}$ and Shaul Mukamel ${ }^{1}$ \\ ${ }^{1}$ Department of Chemistry, University of California, Irvine, California 92697-2025, USA \\ ${ }^{2}$ Center for Nonlinear Phenomena and Complex Systems, Campus Plaine, Code Postal 231, Universitibre de Bruxelles, \\ B-1050 Brussels, Belgium \\ (Received 14 February 2007; published 8 August 2007)
}

\begin{abstract}
The statistical properties of quantum particles moving between two heat reservoirs at different temperatures are studied by solving the quantum master equation using a generating function technique. Bosons and fermions satisfy the same fluctuation theorem: Logarithm of the ratio of the probabilities of forward and backward transports reaches a constant value at long times, with an asymptotic $\sim 1 / t$ correction. Non-Poissonian transfer statistics (bunching for bosons and antibunching for fermions) are examined using the Mandel parameter. These come primarily from the tail of the distribution corresponding to transfer of large number of particles, $k \gg\langle k\rangle$.
\end{abstract}

DOI: 10.1103/PhysRevB.76.085408

PACS number(s): 05.60. $-\mathrm{k}, 44.10 .+\mathrm{i}, 05.30 .-\mathrm{d}$

\section{INTRODUCTION}

With the recent advances in technology, it is now possible to construct submicron (mesoscopic) electronic devices with fairly controlled physical parameters. Fluctuations play a fundamental role in transport through such small devices. Quantum effects and the statistical properties of the current carrying particles dominate the transport properties. This has led to several recent experimental ${ }^{1-6}$ and theoretical ${ }^{7-11}$ studies devoted to quantum transport statistics of current carrying particles (fermions and bosons) through mesoscopic junctions. Most experimental studies employ the Hanbury, Brown, and Twiss ${ }^{12}$ (HBT) configuration where an incoming particle current (beam) is split into two beams and the crosscovariance between the two output beams provides information about the quantum nature of the source.

In Ref. 3, a HBT-type experiment was conducted on a thermal source of two-dimensional electrons and a negative cross-covariance (antibunching) was directly observed between the two electron beams. This was analyzed later theoretically by Texier and Büttiker. ${ }^{8}$ They predicted that, within certain parameter range, a positive cross-covariance (bunching) can also be observed due to inelastic scattering between different current carrying states. This has recently been confirmed experimentally. ${ }^{2}$

Theoretical analysis of the photon counting statistics of a laser-driven molecule has been done earlier. ${ }^{13-17}$ HBT experiments were also carried out on ultracold bosonic atoms. Full counting statistics of a monoenergetic atom laser obtained from the Bose-Einstein condensate of ${ }^{87} \mathrm{Rb}$ atoms has been carried out. ${ }^{18}$ Jeltes et al. ${ }^{19}$ have reported a comparison of the fermionic and the bosonic HBT effect on thermal ${ }^{3} \mathrm{He}$ (fermion) and ${ }^{4} \mathrm{He}$ (boson) atoms. Most HBT experimental studies focus only on the second-order moment of the particle counting statistics which can be more easily sampled. In order to have a full understanding of transport properties of a quantum device, one needs to understand the full probability distribution, which involves all moments. Öttl et al. ${ }^{18}$ have reported the full counting statistics of the atom laser which was found to be Poissonian. However, a systematic comparative study (experimental or theoretical) of full counting sta- tistics of bosons and fermions is still lacking. This is the motivation for the present work.

We shall use a quantum master equation ${ }^{21,20}$ (QME) to compare the statistics of boson and fermion transfers through a mesoscopic system attached to two baths with different temperatures. We compare the full counting statistics of the forward and the backward transfer processes in a nonequilibrium steady state. We observe super (sub)-Poissonian behavior in the transport statistics of bosons (fermions) across the junction. Deviations of the probability distribution functions (PDFs) for the bosons and fermions from Poisson form arise mainly from large (rare) fluctuations $(k \gg\langle k\rangle$, where $k$ is the number of particles transferred in a given time). We also compared the steady-state fluctuation theorem ${ }^{22,23}$ for bosons and fermions and found that bosons with a temperature driving force satisfy a fluctuation theorem very similar to fermions driven by a chemical-potential gradient. ${ }^{24}$ For finite times, deviations from the fluctuation theorem (FT) decay asymptotically as $1 / t$, irrespective of particle statistics.

In the next section, we briefly discuss the QME for a boson system attached to two harmonic baths and obtain the PDF using a generating function technique. We compute the Mandel parameter $M$ (or the Fano factor, $F=M+1$ ) which is directly obtained from the HBT-type experiments. The fluctuation theorem for fermions and bosons is also presented. In Sec. IV, we numerically compare the two statistics. We conclude in Sec. V.

\section{QME FOR BOSON TRANSPORT}

We consider a model of noninteracting bosons which can be transferred between two baths held at two different temperatures, $T_{l}$ (left) and $T_{r}$ (right), through a single site (the system) with energy $\omega_{0}(\hbar=1)$. The model can represent, for example, phonons and the system could be a single harmonic vibration. The total Hamiltonian of the system is

$$
H=\omega_{0} b^{\dagger} b+\sum_{i \in \nu} \omega_{i} a_{i}^{\dagger} a_{i}+\sum_{i \nu} \kappa_{i}^{\nu}\left(a_{i \nu}^{\dagger}+a_{i \nu}\right)\left(b^{\dagger}+b\right),
$$


where $b^{\dagger}(b)$ are the boson creation (annihilation) operators at the site. The other two terms represent the free baths and the bath-system coupling, respectively. $\kappa_{i}^{\nu}$ is the coupling between the system and the $i$ th oscillator of the $\nu$ th $(\nu$ $=l, r)$ bath. $a^{\dagger}(a)$ are the bath boson creation (annihilation) operators. These operators satisfy commutation relation

$$
\psi_{i} \psi_{j}^{\dagger}-\psi_{j}^{\dagger} \psi_{i}=\delta_{i j}, \quad \psi_{i}=b, a_{i \nu} .
$$

The two baths have different temperatures and the system population dynamics will be described by a master equation. We assume that the system-bath coupling is weak so that it can be treated by second-order perturbation and the bath correlation time scale is small compared to the time scale of evolution of the system density matrix. The time dependence of the system density matrix can be described by the QME of the Lindblad form ${ }^{25,26}$

$$
\begin{aligned}
\frac{\partial}{\partial t} \rho^{n}(t)= & -i \omega_{0}\left[b^{\dagger} b, \rho^{n}(t)\right]+\left[\bar{k}_{u} b^{\dagger} \rho^{n-1}(t) b+\bar{k}_{d} b \rho^{n+1}(t) b^{\dagger}\right. \\
& \left.-\bar{k}_{d} \rho^{n}(t) b^{\dagger} b-\bar{k}_{u} b b^{\dagger} \rho^{n}(t)+\text { H.c. }\right],
\end{aligned}
$$

where $\rho^{n}$ represents the system density matrix with $n$ number of quanta on the system. Note that in Eq. (3), coherences between system many-body states with different numbers of quanta do not contribute. ${ }^{27} \bar{k}_{d}\left(\bar{k}_{u}\right)$ is the transfer rate of quanta out of (into) the system and is given in terms of the bath correlation functions

$$
\begin{aligned}
& \bar{k}_{d}=\sum_{i \nu}\left|\kappa_{i}^{\nu}\right|^{2} \int_{0}^{\infty} d \tau e^{i \omega_{0} \tau}\left\langle B_{i \nu} B_{i \nu}^{\dagger}(\tau)\right\rangle, \\
& \bar{k}_{u}=\sum_{i \nu}\left|\kappa_{i}^{\nu}\right|^{2} \int_{0}^{\infty} d \tau e^{-i \omega_{0} \tau}\left\langle B_{i \nu}^{\dagger} B_{i \nu}(\tau)\right\rangle,
\end{aligned}
$$

where $B_{i \nu}=a_{i \nu}^{\dagger}+a_{i \nu}$ and $\langle\cdots\rangle$ represents trace over the bath equilibrium density matrix. The contributions of the two baths to the rates are additive and we have $\bar{k}_{d}=\bar{k}_{d}^{l}+\bar{k}_{d}^{r}$ and $\bar{k}_{u}=\bar{k}_{u}^{l}+\bar{k}_{d}^{r}$.

By plugging the $n$th eigenstate $|n\rangle$ from the left and the right to Eq. (3) and using $b|n\rangle=\sqrt{n}|n-1\rangle$ and $b^{\dagger}|n\rangle$ $=\sqrt{n+1}|n+1\rangle$, we obtain ${ }^{20}$

$\frac{\partial \mathcal{P}_{n}}{\partial t}=n k_{u} \mathcal{P}_{n-1}(t)-\left[n k_{d}+(n+1) k_{u}\right] \mathcal{P}_{n}(t)+(n+1) k_{d} \mathcal{P}_{n+1}(t)$,

where $\mathcal{P}_{n}$ is the probability of having $n(=0,1, \ldots, \infty)$ quanta in the system, with $k_{d}=\bar{k}_{d}+\bar{k}_{d}^{*}$ and $k_{u}=\bar{k}_{u}+\bar{k}_{u}^{*}$. By calculating the bath correlation functions, we obtain $k_{d}=\Gamma\left(\omega_{0}\right)[1$ $\left.+\bar{n}\left(\omega_{0}\right)\right]$ and $k_{u}=\Gamma\left(\omega_{0}\right) \bar{n}\left(\omega_{0}\right)$, where $\bar{n}_{\nu}\left(\Omega_{0}\right)=\left(e^{\beta_{\nu} \omega_{0}}-1\right)^{-1}$ is the Bose-Einstein distribution for the bath with $\beta_{\nu}$ $=1 / k_{B} T_{\nu}, \nu=l, r$ and $\Gamma\left(\omega_{0}\right)=\Sigma_{i \nu}\left|\kappa_{i}^{\nu}\right|^{2}\left(\omega_{0} / \Omega_{i}^{\nu}\right) \delta\left(\Omega_{i}^{\nu}-\omega_{0}\right)$.

Note that for each bath the two rates are related as

$$
k_{d}^{\nu}=e^{\omega_{0} \beta_{\nu}} k_{u}^{\nu}, \quad \nu=l, r
$$

and therefore

$$
\frac{k_{d}^{l} k_{u}^{r}}{k_{u}^{l} k_{d}^{r}}=e^{-\omega_{0} \Delta \beta}
$$

where $\Delta \beta=\beta_{r}-\beta_{l}$. Equation (8) will be crucial for proving the FT for boson transfer.

\section{GENERATING FUNCTION FOR BOSON COUNTING STATISTICS}

The statistics of thermal fluctuations is related to the number $(k)$ of quanta transferred between the baths and the system. The probability $P(k, t)$ that $k$ quanta are transferred during time $t$ is

$$
P(\mathbf{k}, t)=\left\langle\hat{I} \mid \hat{\rho}^{(k)}(t)\right\rangle,
$$

where $\hat{I}$ is the identity matrix and $\hat{\rho}^{(k)}(t)$ is the system density-matrix conditional that $\mathbf{k}$ quanta transferred between the bath and the system (see Appendix A). In the following, we shall denote a matrix, a vector, and a scalar by $\hat{B},|\hat{B}\rangle$, and $B$, respectively. The generating (characteristic) function (GF) for the probability $P(\mathbf{k}, t)$ is defined as

$$
G(\lambda, t)=\sum_{\mathbf{k}} P(\mathbf{k}, t) e^{\lambda \cdot \mathbf{k}}
$$

Since there are four possible elementary processes through which the number of particles (heat quanta) in the system can change (either "in" or "out" from left and right junctions), $\lambda \cdot \mathbf{k}=\sum_{i=1}^{4} \lambda_{i} k_{i}$, where $\mathbf{k}=\left\{k_{i}\right\}, i=1,4$, and $\lambda=\left\{\lambda_{i}\right\}$ are four-component vectors with $k_{2}\left(k_{1}\right)\left[k_{4}\left(k_{3}\right)\right]$ representing the number of quanta transferred from (to) the left (right) bath to (from) the system at time $t$.

All observables of interest can be calculated by taking the derivative of the GF with respect to $\lambda_{i}$. For example, the average number of quanta transferred through process $i$ at time $t$,

$$
\left\langle k_{i}\right\rangle(t)=\sum_{\mathbf{k}} k_{i} P(\mathbf{k}, t)=\left.\frac{\partial}{\partial \lambda_{i}}\langle\hat{I} \mid \hat{G}(\lambda, t)\rangle\right|_{\lambda=0} .
$$

The GF will be calculated for the model of Eq. (6) by solving the equation of motion (see Appendix A for derivation)

$$
|\dot{\hat{G}}(\lambda, t)\rangle=\hat{\mathcal{M}}(\lambda)|\hat{G}(\lambda, t)\rangle,
$$

where $|\hat{G}(\lambda, t)\rangle$ is an infinite dimensional vector and $\hat{\mathcal{M}}$ is a tridiagonal matrix 


$$
\hat{\mathcal{M}}=\left(\begin{array}{ccccccc}
-k_{u} & k_{d}^{l} e^{\lambda_{1}}+k_{d}^{r} e^{\lambda_{3}} & 0 & \ldots & \ldots & \ldots & \ldots \\
k_{u}^{l} e^{\lambda_{2}}+k_{u}^{r} e^{\lambda_{4}} & -\left[k_{d}+2 k_{u}\right] & 2\left(k_{d}^{l} e^{\lambda_{1}}+k_{d}^{r} e^{\lambda_{3}}\right) & 0 & \ldots & \ldots & \ldots \\
0 & 2\left(k_{u}^{l} e^{\lambda_{2}}+k_{u}^{r} e^{\lambda_{4}}\right) & -\left[2 k_{d}+3 k_{u}\right] & 3\left(k_{d}^{l} e^{\lambda_{1}}+k_{d}^{r} e^{\lambda_{3}}\right) & 0 & \ldots & \ldots \\
\ldots & \ldots & \ldots & \ldots & \ldots & \ldots & \ldots \\
\ldots & \ldots & \ldots & \ldots & \ldots & \ldots & \ldots
\end{array}\right),
$$

with $|\hat{G}(\lambda, t=0)\rangle=|\hat{\rho}(0)\rangle$. The formal solution of Eq. (12) is

$$
|\hat{G}(\lambda, t)\rangle=e^{\hat{\mathcal{M}}(\lambda) t}|\hat{\rho}(0)\rangle .
$$

It can be expanded in the left and right eigenvectors $\left\langle\hat{L}_{\xi}(\lambda)\right|$ and $\left|\hat{R}_{\xi}(\lambda)\right\rangle$ of $\hat{\mathcal{M}}(\lambda)$ corresponding to the eigenvalue $\gamma_{\dot{\xi}}(\lambda)$,

$$
|\hat{G}(\lambda, t)\rangle=\sum_{m=0} e^{\gamma_{\xi}(\lambda) t}\left|\hat{R}_{\xi}(\lambda)\right\rangle\left\langle\hat{L}_{\xi}(\lambda) \mid \hat{\rho}(0)\right\rangle .
$$

Note that in order for the system to reach a steady state, the real part of all the eigenvalues $\gamma_{\xi}(\lambda=0),(\xi \neq 0)$ must be negative and $\gamma_{0}(\lambda=0)=0$. The standard method to solve tridiagonal matrix equations, such as Eq. (12), is to use the continued fraction method. ${ }^{14,28}$ Instead, we shall solve Eq. (15) numerically.

\section{A. Average current and the Mandel parameter}

We first consider the lowest two moments of the current. The average number of bosons transferred between the system and the baths through a process $(i=1,2,3,4)$ during time $t$ is given by the derivative of the GF with respect to the corresponding parameter $\lambda_{i}$ at $\lambda=0$, as given in Eq. (11). The average heat $Q_{a v}$ transferred between the system and the bath is therefore $Q_{a v}^{i}(t)=\omega_{0}\left\langle k_{i}\right\rangle(t)$. The corresponding current $I_{i}(t)$ is given by the rate of change of the average number of quanta transferred,

$$
I_{i}(t)=\frac{\partial}{\partial t}\left\langle k_{i}\right\rangle(t) .
$$

At steady state, $\left\langle k_{i}\right\rangle$ grows linearly with time. By differentiating Eq. (14) with respect to $\lambda_{i}$, we get

$$
\left.\frac{\partial}{\partial \lambda_{i}}|\hat{G}(\lambda, t)\rangle\right|_{\lambda=0}=\left.t \frac{\partial \hat{\mathcal{M}}(\lambda)}{\partial \lambda_{i}} e^{\hat{\mathcal{M}}(\lambda) t}\right|_{\lambda=0}|\hat{\rho}(0)\rangle .
$$

In the long-time limit, $e^{\hat{\mathcal{M}}(\lambda=0) t}|\hat{\rho}(0)\rangle=\left|\hat{\rho}_{s t}\right\rangle$. This vector corresponds to the stationary-state solution of Eq. (6). Equation (17) with Eq. (11) then gives

$$
\left\langle k_{i}\right\rangle(t)=\left\langle\hat{I}\left|\hat{\Gamma}_{i}\right| \hat{\rho}_{s t}\right\rangle t,
$$

where $\hat{\Gamma}_{i}=\partial \hat{\mathcal{M}}(\lambda) /\left.\partial \lambda_{i}\right|_{\lambda=0}$ is known as the resetting matrix ${ }^{29}$ for the $i$ th process. The stationary solution of Eq. (6) is ${ }^{20}$

$$
\rho_{s t}^{n}=\frac{\left(k_{d}-k_{u}\right)}{k_{d}}\left(\frac{k_{u}}{k_{d}}\right)^{n} .
$$

The steady-state current $I_{i}^{S}$ is

$$
I_{i}^{S}=\lim _{t \rightarrow \infty} \frac{1}{t}\left\langle k_{i}\right\rangle(t)=\left.\lim _{t \rightarrow \infty} \frac{1}{t} \frac{\partial}{\partial \lambda_{i}} G(\lambda, t)\right|_{\lambda=0}=\left\langle\hat{I}\left|\hat{\Gamma}_{i}\right| \hat{\rho}_{s t}\right\rangle .
$$

Substituting Eq. (19) into Eq. (20), we obtain

$$
\begin{gathered}
I_{1(3)}^{\nu}=\frac{k_{d}^{\nu} k_{u}}{k_{d}-k_{u}}, \quad \nu=l, r, \\
I_{2(4)}^{\nu}=\frac{k_{u}^{\nu} k_{d}}{k_{d}-k_{u}} .
\end{gathered}
$$

The total steady-state current from the left (right) bath to the system is $I^{S}=I_{2(4)}^{\nu}-I_{1(3)}^{\nu}$.

All other moments and cumulants of the probability distribution function can be obtained from the GF by taking higher-order derivatives with respect to $\lambda$. The second cumulant for the $i$ th transport process, $\mathcal{C}_{i}^{(2)}=\left\langle k_{i}^{2}\right\rangle-\left\langle k_{i}\right\rangle^{2}$, can be obtained as

$$
\mathcal{C}_{i}^{(2)}(t)=\left.\frac{\partial^{2}}{\partial \lambda_{i}^{2}} \ln G(\lambda, t)\right|_{\lambda=0} .
$$

The Mandel parameter ${ }^{30}$ for the $i$ th process $M_{i}$ is defined as

$$
M_{i}(t) \equiv \frac{\mathcal{C}_{i}^{(2)}(t)}{\left\langle k_{i}\right\rangle(t)}-1
$$

For Poisson statistics, $M(t)=0 . M>0$ and $M<0$ correspond to super-Poissonian (bunching) and sub-Poissonian (antibunching) statistics. The Mandel parameter has been widely used $^{3,31}$ as a convenient measure of non-Poissonian statistics. The Fano factor, ${ }^{32} F_{i} \equiv M_{i}+1$, is sometimes used ${ }^{3,33,34}$ instead. Assuming that the initial state $\rho(0)$ (when counting starts) corresponds to the steady state, the Mandel parameter can be expressed ${ }^{14,24}$ in terms of the eigenvalues $\left(\gamma_{\xi}\right)$ and the left and right eigenvectors, $\left\langle\hat{L}_{\xi}\right|$ and $\left|\hat{R}_{\xi}\right\rangle$, of $\mathcal{M}(\lambda=0)$.

$$
M_{i}(t)=2 \sum_{\xi},\left(\frac{e^{\gamma_{\xi} t}-1}{\gamma_{\xi} t}+\frac{1}{\gamma_{\xi}}\right) \frac{\left\langle\hat{L}_{0}\left|\hat{\Gamma}_{i}\right| \hat{R}_{\xi}\right\rangle\left\langle\hat{L}_{\xi}\left|\hat{\Gamma}_{i}\right| \hat{R}_{0}\right\rangle}{\left\langle\hat{L}_{0}\left|\hat{\Gamma}_{i}\right| \hat{R}_{0}\right\rangle},
$$

where $\left\langle\hat{L}_{0}\right|$ and $\left|\hat{R}_{0}\right\rangle$ are the eigenvectors corresponding to zero eigenvalue $\left(\gamma_{0}=0\right)$ and the prime indicates that the sum over $\xi$ excludes the zero eigenvalue.

\section{B. Steady-state fluctuation theorem for bosons}

The net heat current flowing through the left and the right system-bath interfaces can be calculated by substituting $\lambda_{1}$ 
$=-\lambda_{2} \equiv \lambda_{L}$ and $\lambda_{4}=-\lambda_{3} \equiv \lambda_{R}$ in the generating function $G(\lambda, t)$. The average current through each junction is then computed by taking the derivative of the current GF with respect to $\lambda_{L}$ or $\lambda_{R}$. At steady state, the two currents are the same and given by the sum of the currents defined in Eqs. (21).

The steady-state FT for currents quantifies the probability of the forward and backward transfer processes. It follows from Eq. (8), as shown in Appendix B, that

$$
\lim _{t \rightarrow \infty} \frac{P(k, t)}{P(-k, t)}=e^{\omega_{0} \Delta \beta k},
$$

where $k$ is the net number of transfer events between the $\nu$ th $(\nu=l, r)$ bath and the system over a time $t$. According to Eq. (25), the probability of transferring bosons against the heat gradient is exponentially small compared to that of the reverse process. The fraction of reverse processes is suppressed exponentially as the system frequency, temperature gradient, or the number of transfers $k$ is increased.

\section{Comparison with fluctuation theorem of fermions}

Equation (3) has the same structure as for a Fermi system. ${ }^{27}$ The difference arises from the commutation rules (boson statistics) of the system and bath operators (see Appendix C).

We consider a system with a single orbital with energy $\epsilon_{0}$ attached to two metal leads. We assume that the leads are at equilibrium with their respective temperatures $T_{l}$ and $T_{r}$ and chemical potential $\mu_{0}$. The statistics of electron transport through a system of coupled quantum dots has recently been studied $^{24}$ using the QME. Here, we extend that model by incorporating the temperature gradient between the two leads. Following Ref. 24, the steady-state FT for this model reads
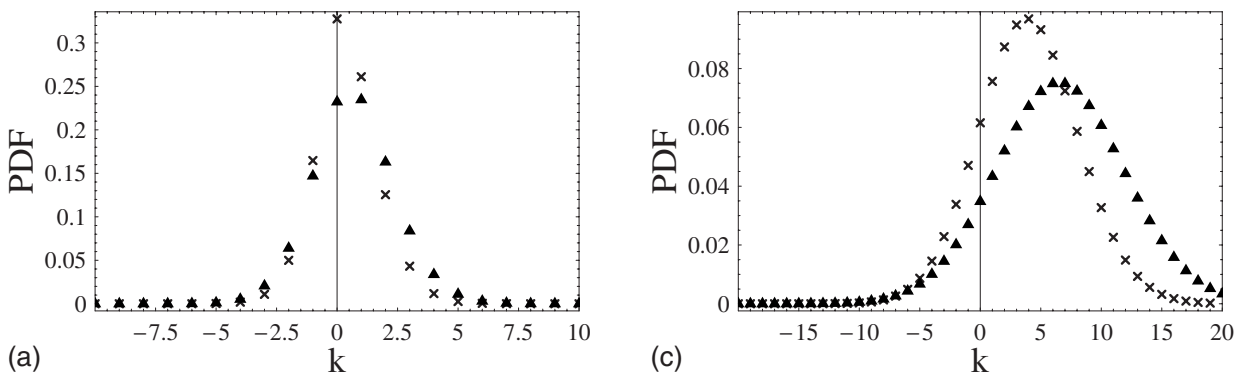

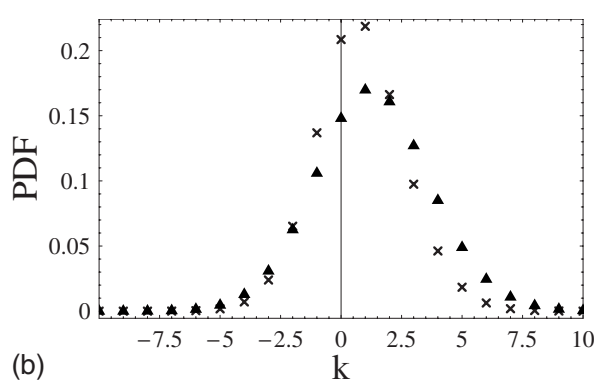

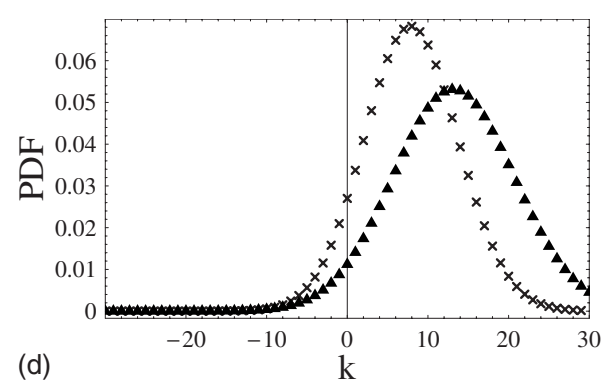

$085408-4$

$$
\lim _{t \rightarrow \infty} \frac{P(k, t)}{P(-k, t)}=e^{\left(\epsilon_{0}-\mu_{0}\right) k \Delta \beta} .
$$

Note that when $\epsilon_{0}=\mu_{0}$, the ratio of the two PDFs is unity and the system is always at equilibrium irrespective of $\Delta \beta$.

The fluctuation theorems (25) and (26) only hold for large time. In Ref. 24, it was shown that for finite times and small nonequilibrium constraints, when the PDF can be well approximated by a Gaussian function, the FT modifies to

$$
\frac{P(k, t)}{P(-k, t)}=e^{(\mathcal{S}-\alpha(t)) k},
$$

and the GF satisfies the symmetry

$$
G(\lambda, t)=G(\mathcal{S}-\lambda-\alpha(t), t),
$$

where $\mathcal{S}=\omega_{0} \Delta \beta$ for bosons, $\mathcal{S}=\left(\epsilon_{0}-\mu_{0}\right) \Delta \beta$ for fermions, and $\alpha(t)$ is the finite time correction (FTC) which $\alpha(t) \rightarrow 0$ as $t \rightarrow \infty$. Since $G(\lambda=0, t)=1$, Eq. (27) reduces to

$$
G(\mathcal{S}-\alpha(t), t)=1 \text {. }
$$

At sufficiently long times (after few tens of transfer events), $\alpha(t)=\alpha_{X} / t$ and Eq. (28) can be solved ${ }^{24}$ to compute the FTC for bosons $\left(\alpha_{X}=\alpha_{b}\right)$ and fermions $\left(\alpha_{X}=\alpha_{f}\right)$. For the transfer statistics between the system and left bath, we obtained

$$
\begin{aligned}
& \alpha_{b}=\frac{1}{I^{s}} \log \left[\frac{\left(k_{d}-k_{u}\right)^{2} k_{u}^{r} k_{d}^{r}}{\left(k_{d}^{r}-k_{u}^{r}\right)\left(k_{d}^{2} k_{u}^{r}-k_{u}^{2} k_{d}^{r}\right)}\right], \\
& \alpha_{f}=\frac{1}{I_{f}^{s}} \log \left[\frac{\left(v_{d}^{r}+v_{u}^{r}\right)\left(v_{d}^{2} v_{u}^{r}+v_{u}^{2} v_{d}^{r}\right)}{v_{d}^{r} v_{u}^{r}\left(v_{d}+v_{u}\right)^{2}}\right],
\end{aligned}
$$

where $I^{s}$ and $I_{f}^{s}$ are the net steady-state currents for the boson [Eqs. (20)] and fermion ${ }^{27}$ systems, respectively. $v_{u}=\Sigma_{\nu} v_{u}^{\nu}$ and $v_{d}=\Sigma_{\nu} v_{d}^{\nu}$ are the rates corresponding to in and out transfers of fermions from the system. ${ }^{24,27}$ The FTC for the statistics between the right bath and the system is obtained by
FIG. 1. Probability distribution function for boson (cross) and fermion (triangle) transfers between left lead and the system at four different binning times $t$ : (a) 500, (b) 1000, (c) 5000, and (d) 10000 . $\omega_{0}=\epsilon_{0}=2.0$, temperature of two baths is $T_{l}=0.7$ and $T_{r}=0.6$, and the coupling to the left and the right baths is $\kappa_{l}=0.1$ and $\kappa_{r}$ $=0.05$. 

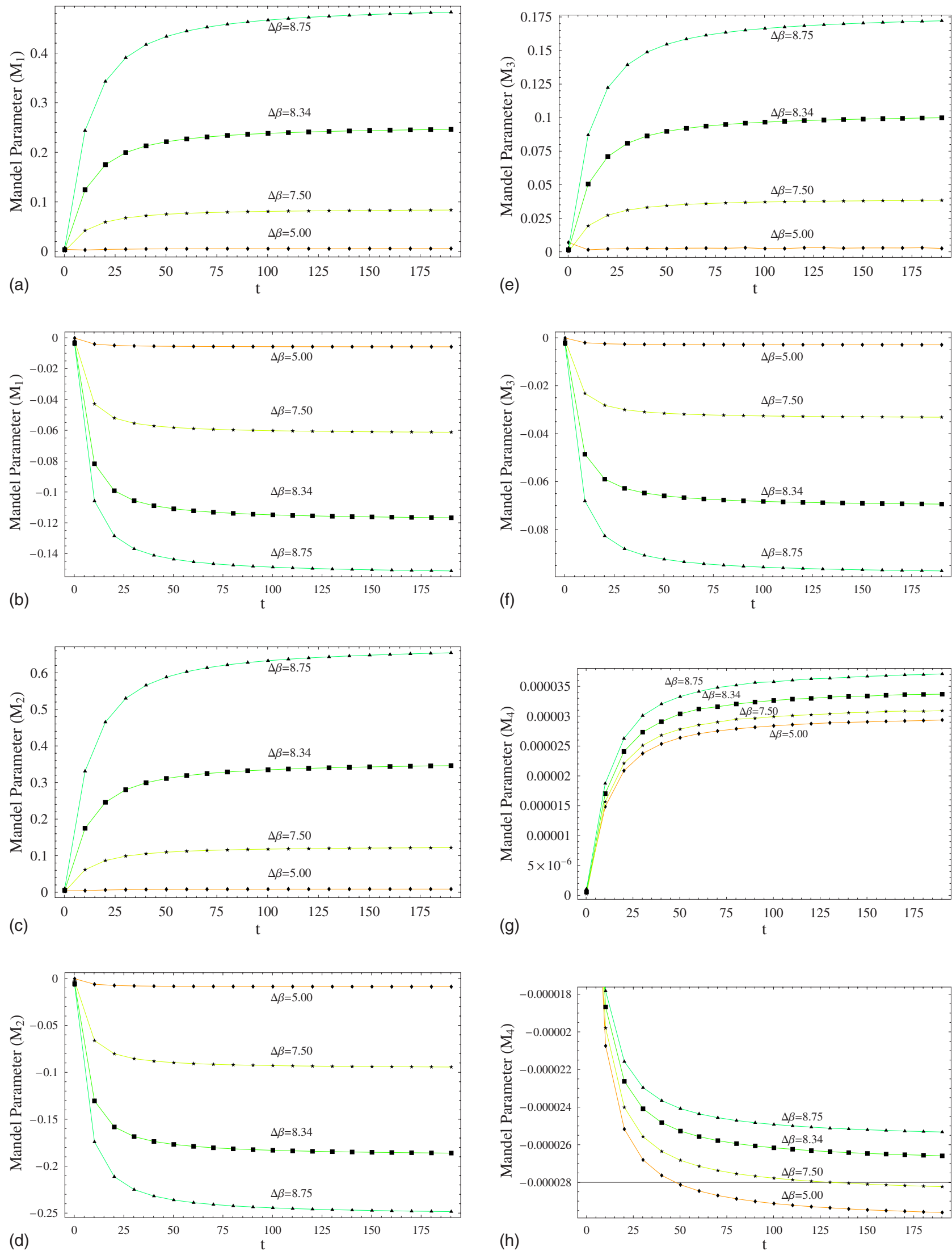

FIG. 2. (Color online) The time-dependent Mandel parameter corresponding to the statistics of (a,c,e,g) boson and (b,d,f,h) fermion transfers. $M_{1}$ and $M_{3}$ correspond to the particle transfer from the system to the left and the right baths while $M_{2}$ and $M_{4}$ correspond to transfer from the left and right baths to the system, respectivel. $\omega_{0}=\epsilon_{0}=1.0, T_{r}=0.1$. 
replacing $\left\{k_{u}^{r}, k_{d}^{r}\right\} \leftrightarrow\left\{k_{u}^{l}, k_{d}^{l}\right\}$ and $\left\{v_{u}^{r}, v_{d}^{r}\right\} \leftrightarrow\left\{v_{u}^{l}, v_{d}^{l}\right\}$ in Eqs. (29) and (30).

Closed expressions for the current and the Mandel parameter in a fermionic system were given in Ref. 24. For the present model (with different bath temperatures), we only need to replace $\beta$ by $\beta_{l}$ and $\beta_{r}$ in the left and right rates [Eqs. (3) in Ref. 24]. The numerical results for fermion and boson statistics are presented in the next section.

\section{NUMERICAL SIMULATIONS}

We used the following parameters in our simulations. The coupling of the system with the left and right baths is fixed at $\kappa_{l}=0.1$ and $\kappa_{r}=0.05$. For the system energy, we consider two different values, $\omega_{0}=1$ and $\omega_{0}=2$. The right bath has the lower temperature $\left(T_{l}>T_{r}\right)$. The current GF is obtained from Eq. (15) for boson and fermion transfers, and the corresponding PDFs are obtained using the inverse Fourier transform of Eq. (10). The QME for bosons is an infinite hierarchy in many-body space. In order to solve the QME for bosons [or Eq. (12)], we therefore need to truncate it at some suitable order $(n)$. This is done by analyzing the exact, analytical solution for the steady-state population, Eq. (19). The manybody states with steady-state population less than $10^{-7}$ have been neglected. Note that the population falls off almost exponentially with the many-body states $(n)$. So, the contribution to statistics coming from many-body states with large $n$ can be safely ignored. In the numerical calculations, $n$ was varied between 5 and 15 , depending on the parameter values $\left\{\omega_{0}, T_{l}, T_{r}\right\}$. In Fig. 1, we show the probability distribution functions for boson and fermion transfers between the left bath and the system, respectively. Positive (negative) $k$ correspond to a net transfer from (to) the left bath. The peak position of the PDF moves to higher values as the observation (binning) time is increased. Equation (18) shows that the average number of particles transferred between the left bath and the system grows linearly in time. The slope that gives the average current, which for the parameter values considered here, is always found to be larger for fermions than for bosons. Therefore, the peak of PDF, which is roughly equivalent to the average number of particles transferred $\langle k\rangle$ for fermions, is always at larger $k$.

In Fig. 2, we compare the Mandel parameter for boson and fermion transfer of different processes as function of time and the inverse temperature difference of baths, $\Delta \beta$. The distribution is non-Poissonian in both cases, bunched $\left(M_{i}>0\right)$ for bosons and antibunched $\left(M_{i}<0\right)$ for fermions. For bosons, all the transfer processes become more bunched as $\Delta \beta$ is increased. For fermions, however, the statistics of the process related to particle transfer from the right lead to the system becomes more Poissonian (see Mandel parameter $\left.M_{4}\right)$ as $\Delta \beta$ is increased. In Fig. 3, we show the PDFs for the transfer from the left bath to the system (corresponding Mandel parameter is $M_{2}$ ) for bosons and fermions. The corresponding Poisson distributions, $f(k)=\langle k\rangle^{k} e^{\langle k\rangle} /(k !)$, are shown as well. The non-Poissonian features $\left(M_{i} \neq 0\right)$ mainly comes from the rare events of transfer of a large number of particles $k \gg\langle k\rangle$. Moreover, we notice that for $k \gg\langle k\rangle$, in the case of
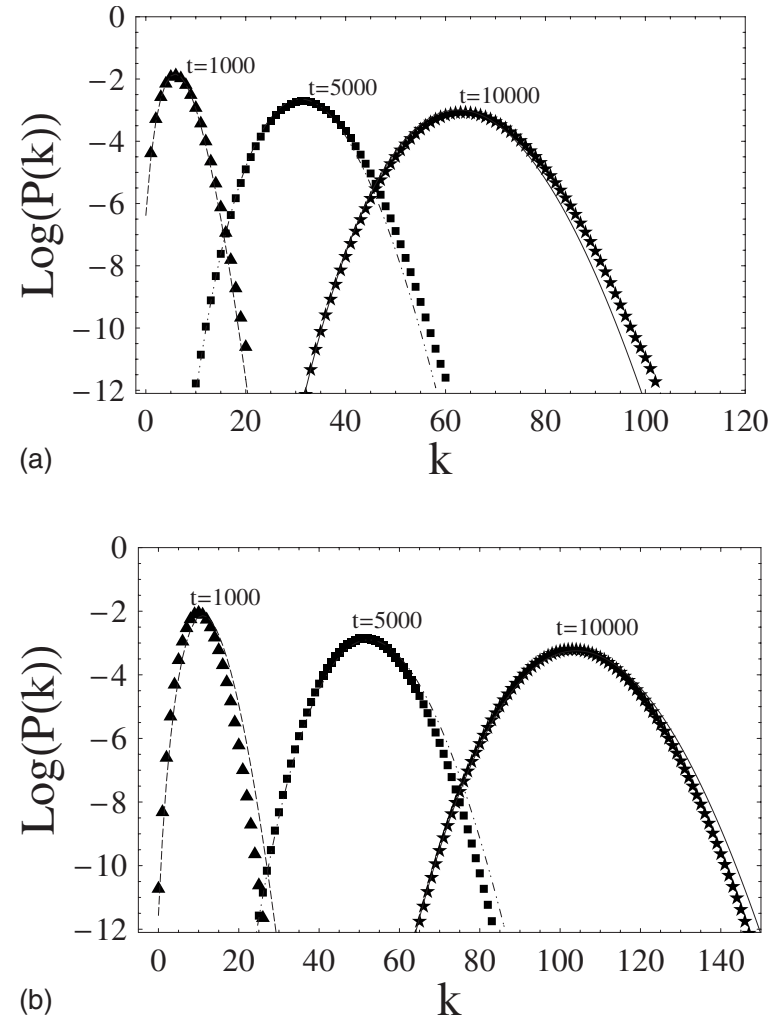

FIG. 3. PDFs corresponding to the (a) boson and (b) fermion transfer processes from the left bath to the system at three different times. Lines represent the Poisson fits (see text). Deviations responsible for a nonzero Mandel parameter are more pronounced at large $k \gg\langle k\rangle$.

bosons (fermions), the Poisson distribution underestimates (overestimates) the actual probability. This is due to the fact that in the case of fermions, due to Pauli exclusion, the system cannot be occupied by more than one particle and the transfer processes are therefore negatively correlated. ${ }^{3}$ This effect shows up more clearly as a large number of particles is transferred. This is why we see a clear deviation from Poisson only for large $k$. For bosons, on the other hand, two or more particles can occupy the same quantum state, enhancing successive transfer events.

By computing the full PDFs for particle transfer statistics, we can compare the fluctuation theorem for bosons and fermions which quantifies the rare events. In the present model, these events correspond to the particle transfer processes against the nonequilibrium constraints. In order to test the fluctuation theorem, we have computed the logarithm of the ratios of the PDF for boson transfer, Eq. (25). The straight line in Fig. 4 represents $\lim _{t \rightarrow \infty} \log [P(k, t) / P(-k, t)]$ as a function of $k$. At finite times, this ratio deviates from the FT, Eq. (26a), which is shown as dots at different times. The deviation from the straight line decreases as the binning time is increased. In Fig. 5, we plot $[\mathcal{S}-\alpha(t)] / \mathcal{S}$ as a function of the binning time $t$. The horizontal line represents the FT. In order to satisfy the FT, more bosons need to be transferred than fermions, i.e., the required binning time for bosons is larger than the fermions. Continuous curves show a fit to 


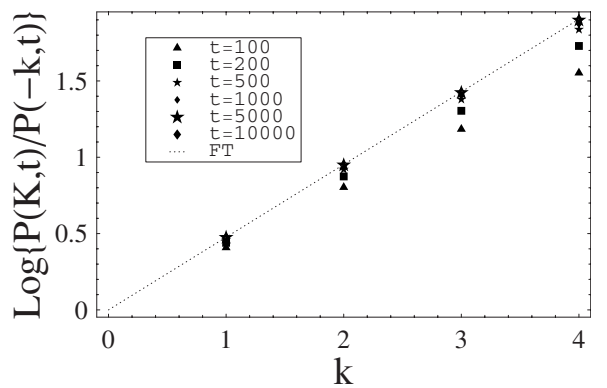

FIG. 4. Fluctuation theorem at finite times for boson statistics. Straight line represents the steady-state FT (25) with slope $\omega_{0} \Delta \beta$ $=0.476$. Dots represent the deviations (slope different from 0.476) for FT at finite times.

$1-\left[\alpha_{b(f)} / \mathcal{S}\right] t^{-1}$, with $\alpha_{b}=8.34$ and $\alpha_{f}=3.97$ for bosons and fermions as obtained by Eqs. (29) and (30), respectively.

\section{A. Interplay of chemical potential and temperature gradient for fermions}

So far, we assumed that the chemical potential of the two baths (leads) is equal. When a finite (dc) bias $(V)$ is applied across the junction such that the two baths are in equilibrium at chemical potentials $\mu_{\nu}, \nu=l, r\left(\mu_{r}=\mu_{0}\right)$, Eq. (26) is modified as

$$
\lim _{t \rightarrow \infty} \frac{P(k, t)}{P(-k, t)}=e^{\left(\epsilon_{0}-\mu_{0}\right) k \Delta \beta} e^{e \beta_{l} \Delta \mu k},
$$

where $\Delta \mu=\mu_{l}-\mu_{r}$ is the applied bias. Note that when $\Delta \beta$ $=0$ or $\epsilon_{0}=\mu_{0}$, Eq. (26) reduces to the result obtained in Ref. 24. The first exponential on the right-hand side of Eq. (26) is due to the heat gradient while the second accounts for the chemical-potential gradient.

According to Eq. (31), when the chemical-potential gradient is opposite $\left(\mu_{l}<\mu_{r}\right)$ to the temperature gradient $\left(\beta_{l}\right.$ $<\beta_{r}$ ) between two leads, for each bias value, there exists a temperature difference $\Delta T=T_{l}-T_{r}$,

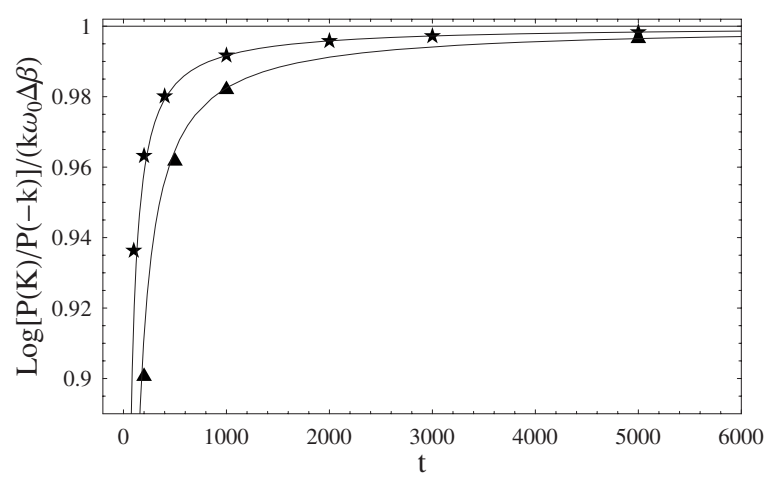

FIG. 5. Time dependence of the finite time correction to FT: $\log [P(k) / P(-k)] /\left(k \omega_{0} \Delta \beta\right)$ is shown at different times. The FT is satisfied asymptotically. Continuous curve is the fit, $1-\alpha_{X} / \mathcal{S t}^{-1}$ with $\alpha_{b}=8.34$ and $\alpha_{f}=3.97$ for bosons and fermions, respectively, as predicted by Eqs. (29) and (30).

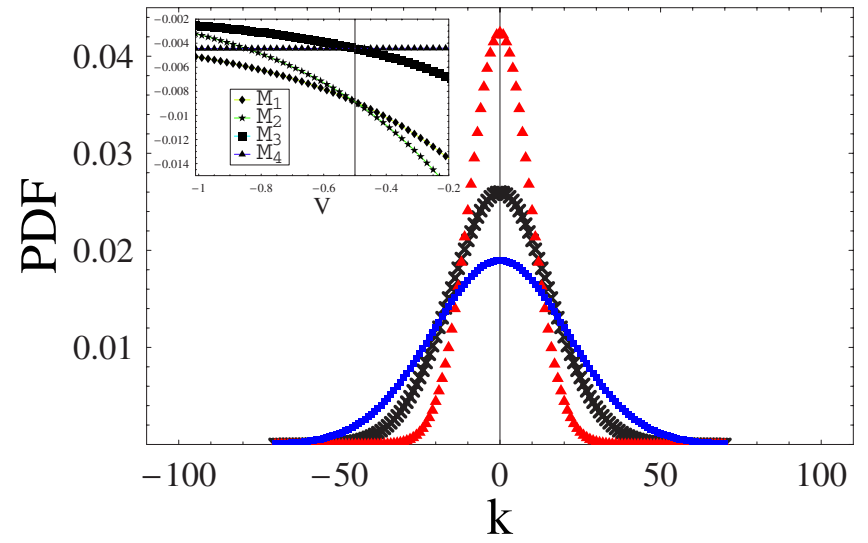

FIG. 6. (Color online) The PDFs for fermions are shown at $t$ $=100$ for three sets of parameters (upper: $T_{l}=0.5, T_{r}=0.4$, and $\Delta \mu=0.5$; middle: $T_{l}=0.8, T_{r}=0.5$, and $\Delta \mu=1.2$; and lower: $T_{l}$ $=0.7, T_{r}=0.6$, and $\left.\Delta \mu=0.34\right)$ when condition (32) is satisfied. Inset shows the Mandel parameters at $t=100$ as a function of the bias with $T_{l}=0.5, T_{r}=0.4$, and $\epsilon_{0}=2$. Note that $M_{1}=M_{2}$ and $M_{3}=M_{4}$ when Eq. (32) holds.

$$
\frac{\Delta T}{T_{r}}=\frac{e V}{\mu_{0}-\epsilon_{0}},
$$

where the ratio of the probabilities in Eq. (26) is unity; the ratio of the rates $v_{d}^{\nu} / v_{u}^{\nu}$ for $\nu=l, r$ becomes equal and the system is at equilibrium. When condition (32) is satisfied, the probability distribution for the current (steady state) is symmetric around $k=0$ and the Mandel parameters corresponding to the transfer process related to the same bath are equal, as shown in Fig. 6. This implies that the two currents $I_{1(3)}^{\nu}$ and $I_{2(4)}^{\nu}$ become equal.

\section{CONCLUSIONS}

We have studied the thermal transport properties of bosons and fermions through a mesoscopic junction. The generating function approach was used to calculate the full counting statistics of the transport. Numerical calculations show that the Mandel parameter for bosons is positive (bunching) while that for fermions, it is negative (antibunching). By computing the full PDFs, we notice that the nonPoissonian character of the statistics mainly comes from the transfer of the large number $(\gg k)$ of particles. We showed that both fermion and boson statistics satisfy a steady-state fluctuation theorem. Irrespective of particle statistics, we found that for finite times, the deviation from the FT, Eqs. (25) and (26), vanishes asymptotically as $1 / t$. This is due to the fact that the leading-order correction to the large deviation function (LDF) defined in Eq. (B6) vanishes as $1 / t$. We further observed that the mean number of particles transferred between the bath and the system grows linearly in time with the value for fermions being larger than that for bosons. 


\section{ACKNOWLEDGMENTS}

The financial support from the National Science Foundation (Grant No. CHE-0446555) and NIRT (Grant No. EEC0406750) is gratefully acknowledged. M.E. is partially supported by the FNRS Belgium (Collaborateur Scientifique).

\section{APPENDIX A: DERIVATION OF EQUATION (12)}

In Liouville space, the QME (3) can be written as

$$
|\dot{\hat{\rho}}(t)\rangle=\hat{\mathcal{W}}|\hat{\rho}(t)\rangle
$$

where $|\hat{\rho}\rangle$ is a vector comprising populations $\rho_{n n}$ and coherences $\rho_{n m}, n \neq m$. Since populations are decoupled from coherences, the matrix Liouville operator $\hat{\mathcal{W}}$ can be factorized in two parts, $\hat{\mathcal{W}}_{c}$ and $\hat{\mathcal{W}}_{p}$, describing the evolution of coherences and the populations, respectively. The off-diagonal elements, $\hat{\Gamma}_{p}$, in $\hat{\mathcal{W}}_{p}$ represent the rate of transfer of particles between the bath and the system and are responsible for the population change due to the particle transfer.

In order to compute the system density-matrix condition $l$ on the transfer of a fixed number of electrons, we transform Eq. (A1) into the interaction picture with respect to $\hat{\mathcal{M}}_{0}$ $\equiv \hat{\mathcal{W}}-\hat{\Gamma}_{p}$.

$$
\left|\dot{\hat{\rho}}_{I}(t)\right\rangle=\hat{\Gamma}_{p}(t)\left|\hat{\rho}_{I}(t)\right\rangle,
$$

where $\left|\hat{\rho}_{I}(t)\right\rangle=\hat{U}(0, t)|\hat{\rho}(t)\rangle$ and $\hat{\Gamma}_{p}(t)=U(0, t) \hat{\Gamma}_{p} U(t, 0)$, with $\hat{U}(0, t)=\exp \left\{-\hat{\mathcal{M}}_{0} t\right\}$, is the time evolution operator in the absence of electron transfer.

The formal solution of Eq. (A1) can be written as

$$
\left|\hat{\rho}_{I}(t)\right\rangle=\exp _{+}\left[\int_{0}^{t} \hat{\Gamma}(\tau) d \tau\right]\left|\hat{\rho}_{I}(0)\right\rangle
$$

Expanding the (time-ordered) exponential in Eq. (A3), we can write $\left|\hat{\rho}_{I}(t)\right\rangle=\Sigma_{k}\left|\hat{\rho}_{I}^{(k)}(t)\right\rangle$, where

$$
\begin{aligned}
\left|\hat{\rho}_{I}^{(k)}(t)\right\rangle= & \int_{0}^{t} d \tau_{k} \int_{0}^{\tau_{k}} d \tau_{k-1} \cdots \\
& \times \int_{0}^{\tau_{2}} d \tau_{1} \hat{\Gamma}_{p}\left(\tau_{k}\right) \hat{\Gamma}_{p}\left(\tau_{k-1}\right) \cdots \hat{\Gamma}_{p}\left(\tau_{1}\right)\left|\hat{\rho}_{I}(0)\right\rangle .
\end{aligned}
$$

In Eq. (A4), each interaction with $\hat{\Gamma}_{p}$ at times $t=\tau_{n}(n$ $=1, k$ ) records the transfer of a particle between baths and the system. Multiplying Eq. (A4) from the left by $\hat{U}(t, 0)$, the conditional density matrix for transferring $k$ particles in the Schrödinger picture is obtained as

$$
\begin{aligned}
\left|\hat{\boldsymbol{\rho}}^{(k)}(t)\right\rangle= & \int_{0}^{t} d \tau_{k} \int_{0}^{\tau_{k}} d \tau_{k-1} \cdots \\
& \times \int_{0}^{\tau_{2}} d \tau_{1} \hat{U}\left(t, \tau_{k}\right) \hat{\Gamma}_{p} \hat{U}\left(\tau_{k}, \tau_{k-1}\right) \hat{\Gamma}_{p} \cdots \\
& \times \hat{U}\left(\tau_{2}, \tau_{1}\right) \hat{\Gamma}_{p} \hat{U}\left(0, \tau_{1}\right)|\hat{\rho}(0)\rangle .
\end{aligned}
$$

According to Eq. (A5) the system (conditional) density matrix evolves from time $t=0$ to $t=\tau_{1}$ without any particle transfer. At $t=\tau_{1}, \hat{\Gamma}_{p}$ acts on the density matrix and a particle is transferred. The density matrix again evolves without any particle transfer until a second particle is transferred at $t$ $=\tau_{2}$. This process is repeated until the $k$ particle has been transferred at $t=\tau_{k}$ and the density matrix finally evolves from $t=\tau_{k}$ to $t=t$ without additional particle transfer. Note that all particle transfers are instantaneous so that the density matrix does not evolve during particle transfer. There are four different processes through which particles can be transferred between the bath and the system, we write $\mathbf{k}$ $=k_{1}, k_{2}, k_{3}, k_{4}$, such that $k=\sum_{i=1}^{4} k_{i}$.

The probability of transferring $\mathbf{k}$ particles at time $t$ is then obtained by tracing the conditional density matrix

$$
P(\mathbf{k}, t)=\left\langle\hat{I} \mid \hat{\rho}^{(k)}(t)\right\rangle=\sum_{i} \int_{0}^{t} d \tau_{k}\left\langle\hat{I}\left|\hat{\Gamma}_{p}^{i}\left(\tau_{k}\right)\right| \hat{\rho}_{I}^{\mathbf{k}-\hat{\mathbb{I}}_{i}}\left(\tau_{k}\right)\right\rangle,
$$

where $\hat{I}_{i}$ is a vector with 1 at the position $i$ and the rest of the elements zero. From Eqs. (10) and (A6), the GF in the interaction picture is then obtained as

$$
\begin{aligned}
\hat{G}_{I}(\lambda, t) & =\sum_{k_{i}, i} \int_{0}^{t} d \tau e^{\lambda_{i} k_{i}}\left\langle\hat{I}\left|\hat{\Gamma}_{p}^{i}(\tau)\right| \hat{\rho}_{I}^{\mathbf{k}-\hat{I}_{i}}(\tau)\right\rangle \\
& =\sum_{i} \int_{0}^{t} d \tau\left\langle\hat{I}\left|e^{\lambda_{i}} \hat{\Gamma}_{p}^{i}(\tau) \sum_{k_{i}} e^{\lambda_{i}\left(k_{i}-1\right)}\right| \hat{\rho}_{I}^{k_{i}-1}(\tau)\right\rangle \\
& =\sum_{i} \int_{0}^{t} d \tau\left\langle\hat{I}\left|e^{\lambda_{i}} \hat{\Gamma}_{p}^{i}(\tau)\right| \hat{G}_{I}(\lambda, \tau)\right\rangle .
\end{aligned}
$$

Taking the time derivative and transforming back to the Schrödinger picture, we get

$$
|\dot{\hat{G}}(\lambda, t)\rangle=\left(\hat{\mathcal{M}}_{0}+\sum_{i} e^{\lambda_{i}} \hat{\Gamma}_{p}^{i}\right)|\hat{G}(\lambda, t)\rangle .
$$

The matrices $\hat{\mathcal{M}}_{0}$ and $\hat{\Gamma}_{p}^{i}$ are defined by the QME (6). When substituting into Eq. (A8), we obtain Eq. (12).

\section{APPENDIX B: DERIVATION OF EQUATION (25)}

Following Ref. 24, Eq. (25) can be derived by defining the LDF for the particle number fluctuations as

$$
R(\zeta) \equiv-\lim _{t \rightarrow \infty} \frac{1}{t} \ln P(\zeta, t),
$$

where $\zeta=k / t$. Equation (B1) uses the ansatz

$$
P(\zeta, t)=C(\zeta, t) e^{-R(\zeta) t},
$$

with $\lim _{t \rightarrow \infty} C(\zeta, t) / t=0$. Using Eqs. (10) and (B2), the current GF can be expressed as

$$
G(\lambda, t)=\int d \zeta C(\zeta, t) e^{-[\lambda \zeta+R(\zeta)] t} .
$$

In long time, the main contribution to the integral in Eq. (B3) comes from the $\zeta=\zeta^{*}$ value where $d R(\zeta) / d \zeta=-\lambda$. Expanding 
$R(\zeta)$ around $\zeta^{*}$ and keeping up to second order and substituting into Eq. (B3), we get

$$
G(\lambda)=e^{-\left[\lambda \zeta^{*}+R\left(\zeta^{*}\right)\right] t} \int d \zeta C(\zeta, t) e^{(1 / 2) \ddot{R}(\zeta)\left(\zeta-\zeta^{*}\right)^{2} t} .
$$

Now consider the generator $\mathcal{M}(\lambda)$ of the current generating operator which is obtained by setting $\lambda_{1}=\lambda=-\lambda_{2}$ and $\lambda_{3}=\lambda_{4}=0$ in Eq. (13). We notice that the $\lambda$-dependent terms in the characteristic polynomial of the current generator appear only as a product, $\mathcal{A}(\lambda) \mathcal{B}(\lambda)=\left(k_{d}^{l} e^{\lambda}+k_{d}^{r}\right)\left(k_{u}^{l} e^{-\lambda}+k_{u}^{r}\right)$. Using Eq. (8), we see that $\mathcal{A}(\lambda) \mathcal{B}(\lambda)=\mathcal{A}\left(-\lambda+\omega_{0} \Delta \beta\right) \mathcal{B}(-\lambda$ $\left.+\omega_{0} \Delta \beta\right)$. Therefore, the characteristic polynomial and hence the eigenvalues $g_{n}(\lambda), n=0,1,2, \ldots$ of the current GF show the symmetry property

$$
g_{n}(\lambda)=g_{n}\left(-\lambda+\omega_{0} \Delta \beta\right) .
$$

We define a LDF, $S(\lambda)$, corresponding to the generating function

$$
S(\lambda)=-\lim _{t \rightarrow \infty} \frac{1}{t} \ln G(\lambda, t) .
$$

Since the eigenvalues of the current GF satisfy the symmetry property (B5), it follows that $S(\lambda)$ also satisfies the same property,

$$
S(\lambda)=S\left(\omega_{0} \Delta \beta-\lambda\right) .
$$

Using Eqs. (B4) and (B6), we obtain

$$
S(\lambda)=\lambda \zeta^{*}+R\left(\zeta^{*}\right) .
$$

Equation (B8) shows that $S(\lambda)$ is the Legendre transform of the LDF $R\left(\zeta^{*}\right)$. The inverse Legendre transform gives $R(\zeta)$ $=S\left(\lambda^{*}\right)-\lambda^{*} \zeta$. Substituting $\lambda^{*}=\omega_{0} \Delta \beta$ into the inverse Legendre transform and using Eq. (B7), we obtain

$$
R(\zeta)=R(-\zeta)-\omega_{0} \zeta \Delta \beta
$$

Using Eqs. (B1) and (B2), it is straightforward to verify Eq. (25).

\section{APPENDIX C: QME FOR FERMIONS}

Here, we present the QME for a fermionic system. The model is a system site coupled to two fermionic (noninteracting) leads held at two different temperatures. The details of the calculations are given in Ref. 27. The Hamiltonian for the system is

$$
H=\epsilon_{0} c_{0}^{\dagger} c_{0}+\sum_{\nu} \epsilon_{\nu} c_{\nu}^{\dagger} c_{\nu}+\sum_{0 \nu}\left(\kappa_{0}^{\nu} c_{0}^{\dagger} c_{\nu}+\text { H.c. }\right),
$$

where $c_{0}^{\dagger}\left(c_{0}\right)$ are the Fermi creation (annihilation) operators for the system site and satisfy the commutation relations $c_{0}^{\dagger} c_{0}+c_{0} c_{0}^{\dagger}=1$. The first two terms in Eq. (C1) represent the noninteracting system and leads; the last term is the coupling between the system and two leads. Direct coupling between the two leads is neglected.

When the coupling $J$ is turned on, the system density matrix evolves according to the QME analogous to Eq. (3).

$$
\begin{aligned}
\frac{\partial}{\partial t} \rho^{n}(t)= & -i \epsilon_{0}\left[c_{0}^{\dagger} c_{0}, \rho^{n}(t)\right]+\left[v_{u} c_{0}^{\dagger} \rho^{n-1}(t) c+v_{d} c_{0} \rho^{n+1}(t) c_{0}^{\dagger}\right. \\
& \left.-v_{d} \rho^{n}(t) c_{0}^{\dagger} c_{0}-v_{u} c_{0} c_{0}^{\dagger} \rho^{n}(t)+\text { H.c. }\right],
\end{aligned}
$$

where $v_{d}=v_{d}^{l}+v_{d}^{r}$ and $v_{u}=v_{u}^{l}+v_{u}^{r}$ are the particle rates

$$
v_{d}^{\nu}=\pi n_{\nu}\left(\epsilon_{0}\right)\left|\kappa_{0}^{\nu}\right|^{2}\left[1-f_{\nu}\left(\epsilon_{0}\right)\right], \quad v_{u}^{\nu}=\pi n_{\nu}\left(\epsilon_{0}\right)\left|\kappa_{0}^{\nu}\right|^{2} f_{\nu}\left(\epsilon_{0}\right),
$$

where $f_{\nu}\left(\epsilon_{0}\right)=\left[1+e^{\beta_{\nu}\left(\epsilon_{0}-\mu_{\nu}\right)}\right]$ is the Fermi function for the $\nu$ th lead at chemical potential $\mu_{\nu}$ and inverse temperature $\beta_{\nu}$. When traced over two system states $|0\rangle$ (empty) and $|1\rangle$ (occupied) in Fock space, and using the relations

$$
\begin{aligned}
& c_{0}|0\rangle=0, \quad c^{\dagger}|0\rangle=|1\rangle, \\
& c_{0}|1\rangle=|0\rangle, \quad c^{\dagger}|1\rangle=0,
\end{aligned}
$$

we obtain the coupled equations

$$
\begin{aligned}
& \dot{\rho}_{0}(t)=-2 v_{u} \rho_{0}(t)+2 v_{d} \rho_{1}(t), \\
& \dot{\rho}_{1}(t)=-2 v_{d} \rho_{1}(t)+2 v_{u} \rho_{0}(t),
\end{aligned}
$$

where $\rho_{n}=\langle n|\hat{\rho}| n\rangle, n=0,1$. Equations (C2) and (C5) are analogous to the boson [Eqs. (3) and (6)], respectively.
${ }^{1}$ T. Rom, Th. Best, D. van Oosten, U. Schneider, S. Folling, B. Paredes, and I. Bloch, Nature (London) 444, 733 (2006).

${ }^{2}$ S. Oberholzer, E. Bieri, C. Schönenberger, M. Giovannini, and J. Faist, Phys. Rev. Lett. 96, 046804 (2006).

${ }^{3}$ W. D. Oliver, J. Kim, R. C. Liu, and Y. Yamamoto, Science 284, 299 (1999).

${ }^{4}$ H. Kiesel, A. Renz, and F. Asselbach, Nature (London) 418, 392 (2002).

${ }^{5}$ M. Iannuzzi, A. Orecchini, F. Sacchetti, P. Facchi, and S. Pascazio, Phys. Rev. Lett. 96, 080402 (2006).

${ }^{6}$ T. Fujisawa, T. Hayashi, R. Tomita, and Y. Hirayama, Science 312, 1634 (2006).

${ }^{7}$ T. L. Schmidt, A. Komnik, and A. O. Gogolin, Phys. Rev. Lett.
98, 056603 (2007).

${ }^{8}$ C. Texier and M. Büttiker, Phys. Rev. B 62, 7454 (2000).

${ }^{9}$ L. S. Levitov and M. Reznikov, Phys. Rev. B 70, 115305 (2004).

${ }^{10}$ L. Basano, P. Ottonello, and B. Torre, J. Opt. Soc. Am. B 22, 1314 (2005).

${ }^{11}$ M. Büttiker, Science 313, 1587 (2006); B. Cleuren and C. Van den Broeck, Europhys. Lett. 79, 30001 (2007).

${ }^{12}$ R. H. Brown and R. Q. Twiss, Nature (London) 177, 27 (1956).

${ }^{13}$ R. J. Glauber, Phys. Rev. 131, 2766 (1963).

${ }^{14}$ F. Sanda and S. Mukamel, J. Chem. Phys. 124, 124103 (2006).

${ }^{15}$ S. Mukamel, Phys. Rev. A 68, 063821 (2003).

${ }^{16}$ L. Mandel, Phys. Rev. Lett. 49, 136 (1982).

${ }^{17}$ F. Sanda and S. Mukamel, Phys. Rev. A 71, 033807 (2005). 
${ }^{18}$ A. Öttl, S. Ritter, M. Köhl, and T. Esslinger, Phys. Rev. Lett. 95, 090404 (2005).

${ }^{19}$ T. Jeltes, J. M. McNamara, W. Hogervorst, W. Vassen, V. Krachmalnicoff, M. Schellekens, A. Perrin, H. Chang, D. Boiron, A. Aspect, and C. I. Westbrook, Nature (London) 445, 402 (2007).

${ }^{20}$ D. Segal and A. Nitzan, Phys. Rev. Lett. 94, 034301 (2005).

${ }^{21}$ D. Segal and A. Nitzan, Phys. Rev. E 73, 026109 (2006).

${ }^{22}$ D. J. Evans, E. G. D. Cohen, and G. P. Morriss, Phys. Rev. Lett. 71, 2401 (1993); D. J. Evans and D. J. Searles, Phys. Rev. E 50, 1645 (1994).

${ }^{23}$ C. Jarzynski, Phys. Rev. Lett. 78, 2690 (1997); Phys. Rev. E 56, 5018 (1997).

${ }^{24}$ M. Esposito, U. Harbola, and S. Mukamel, Phys. Rev. B 75, 155316 (2007).

${ }^{25}$ H.-P. Breuer and F. Petruccione, The Theory of Open Quantum
Systems (Oxford University Press, Oxford, 2002).

${ }^{26}$ C. W. Gardiner and P. Zoller, Quantum Noise (Springer, Berlin, 2000).

${ }^{27}$ U. Harbola, M. Esposito, and S. Mukamel, Phys. Rev. B 74, 235309 (2006).

${ }^{28}$ H. Reskin, The Fokker-Planck Equation (Springer, Berlin, 1989).

${ }^{29}$ G. C. Hegerfeldt, Phys. Rev. A 47, 449 (1993).

${ }^{30}$ L. Mandel, Proc. Phys. Soc. London 74, 236 (1959).

${ }^{31}$ K. Matsuo, M. C. Teich, and B. A. A. Saleh, Appl. Opt. 22, 1898 (1983).

${ }^{32}$ U. Fano, Phys. Rev. 72, 26 (1947).

${ }^{33}$ L. Basano, P. Ottonello, and B. Torre, J. Opt. Soc. Am. B 22, 1314 (2005).

${ }^{34}$ M. C. Teich and B. A. A. Saleh, Opt. Lett. 7, 365 (1982). 